\title{
GUÍA PARA LA EJECUCIÓN DE NEGOCIACIONES
}

\author{
Guide for the execution of negotiations
}

EPISTEMUS

ISSN: 2007-8196 (electrónico)

ISSN: 2007-4530 (impresa)

Daisy Oropesa Méndez

Recibido: 12 de septiembre de 2018 ,

Aceptado: 30 de noviembre del 2018

Autor de Correspondencia:

Daisy Oropesa Méndez

Correo:doropesa@uci.cu

\section{Resumen}

El proceso de negociación está compuesto por varias etapas, considerándose la ejecución entre las más complejas y significativa, ya que es el momento que centra con mayor intensidad la interacción entre las partes negociadoras, y en el que ambas exponen sus intereses y necesidades. Actualmente la bibliografía especializada referencia una serie de modelos de negociación, enunciando los objetivos que deben cubrirse en cada una de sus etapas. Sin embargo, adolecen del detalle requerido para guiar a los especialistas durante la etapa de ejecución de la negociación. Es por ello que la presente investigación tuvo como objetivo el desarrollo de una guía que expusiera las actividades a realizar en la etapa de ejecución de una negociación, así como las entradas, técnicas y herramientas, y salidas involucradas en cada una de éstas. El trabajo realizado se basó fundamentalmente en el análisis de documentación y la observación participativa de la autora en diversos procesos de negociación. La propuesta elaborada guiará a los especialistas durante la ejecución de una negociación.

Palabras clave: negociación, etapas de la negociación, ejecución de la negociación, guía práctica.

\section{Abstract}

The negotiation process is made up of several stages, with implementation being considered among the most complex and significant, as it is the moment when the interaction between the negotiating parties is most intense, and in which both parties present their interests and needs. Currently, the specialized bibliography refers to a series of negotiation models, setting out the objectives to be covered in each of their stages. However, they lack the detail required to guide specialists through the execution stage of the negotiation. For this reason, the objective of this research was to develop a guide setting out the activities to be carried out during the execution stage of a negotiation, as well as the inputs, techniques and tools, and outputs involved in each of these. The work carried out was based fundamentally on the analysis of documentation and the author's participatory observation of various negotiation processes. The proposal drawn up will guide the specialists through the execution of a negotiation.

Keywords: negotiation, negotiation threads, negotiation execution, practical guide. 


\section{INTRODUCCIÓN}

De acuerdo a Fisher, Ury y Patton, 2011; cada vez hay más ocasiones que requieren una negociación, todo el mundo quiere participar en las decisiones que le afectan $y$, por el contrario, cada vez son menos las personas que pueden llegar a aceptar decisiones que han sido tomadas por otros.

Para el dominio de la presente investigación se asumen como etapas dentro del proceso de negociación las siguientes: planeación, ejecución, cierre y seguimiento; tomando como base el estudio de diversos autores que incluyen diferentes actividades que pueden enmarcarse dentro de ésta clasificación (Cano y Baena, 2015), (Ogliastri, 1998), (Anthopoulos y Xristianopoulou, 2012), (Linares, Calderón, Donatti, Hernández y Martínez, 2009), (Mills, 2016), (Zapiola, 2009), (Casal, 2016), (Tarantino-Curseri, 2017).

Dentro de las etapas que conforman el proceso de negociación la ejecución se considera entre las más complejas y significativa, ya que es el momento que centra con mayor intensidad la interacción entre las partes negociadoras. En esta etapa se da el intercambio de información, las partes exponen sus respectivas ofertas y esperan la aceptación de la otra parte. Ésta etapa es significativa porque se exponen las necesidades e intereses por los cuales se acude a la negociación. Aquí se presentarán diferentes estilos y tácticas de negociación que se deberán saber manejar. Además, es sustancial preguntar para conocer a exactitud lo que desea conseguir la otra parte y cuanto están dispuestos a ceder a fin de llevar la negociación por buen camino (Fisher, Ury y Patton, 2011).

Actualmente la bibliografía especializada referencia una serie de modelos de negociación, enunciando los objetivos que deben cubrirse en cada una de sus etapas. Sin embargo, adolecen del detalle requerido para guiar a los especialistas durante la etapa de ejecución de la negociación. Es por ello que la presente investigación se centra en la propuesta de una guía que exponga las actividades a realizar en la etapa de ejecución de una negociación, así como las entradas, técnicas y herramientas, y salidas involucradas en cada una de éstas.

\section{MATERIALES Y MÉTODOS O METODOLOGÍA COMPUTACIONAL}

Para la ejecución de la presente investigación se utilizó el método analítico-deductivo y como técnica de recolección de datos el análisis documental. Se realizó el análisis de la literatura especializada publicada a nivel nacional e internacional, y se empleó la observación participativa de la autora en diversos procesos de negociación.

Durante la etapa de ejecución de la negociación; se propone la realización de las tres actividades que se exponen a continuación.

\section{Actividad 1. Presentación de la oferta.}

Esta actividad marcará la apertura de la negociación con la contraparte negociadora. Durante su ejecución debe prestarse mucha atención al ambiente, ya que éste usualmente define el tono de toda la negociación (Antonio y Barraza, 2017). Después de haber creado el ambiente más adecuado a los objetivos y estrategias de la negociación, se inicia la presentación de acuerdo al plan previamente elaborado. Es en este momento donde la comunicación comienza a jugar un importante rol.

La comunicación es tan antigua como el ser humano. El hombre y la mujer son comunicadores por naturaleza;

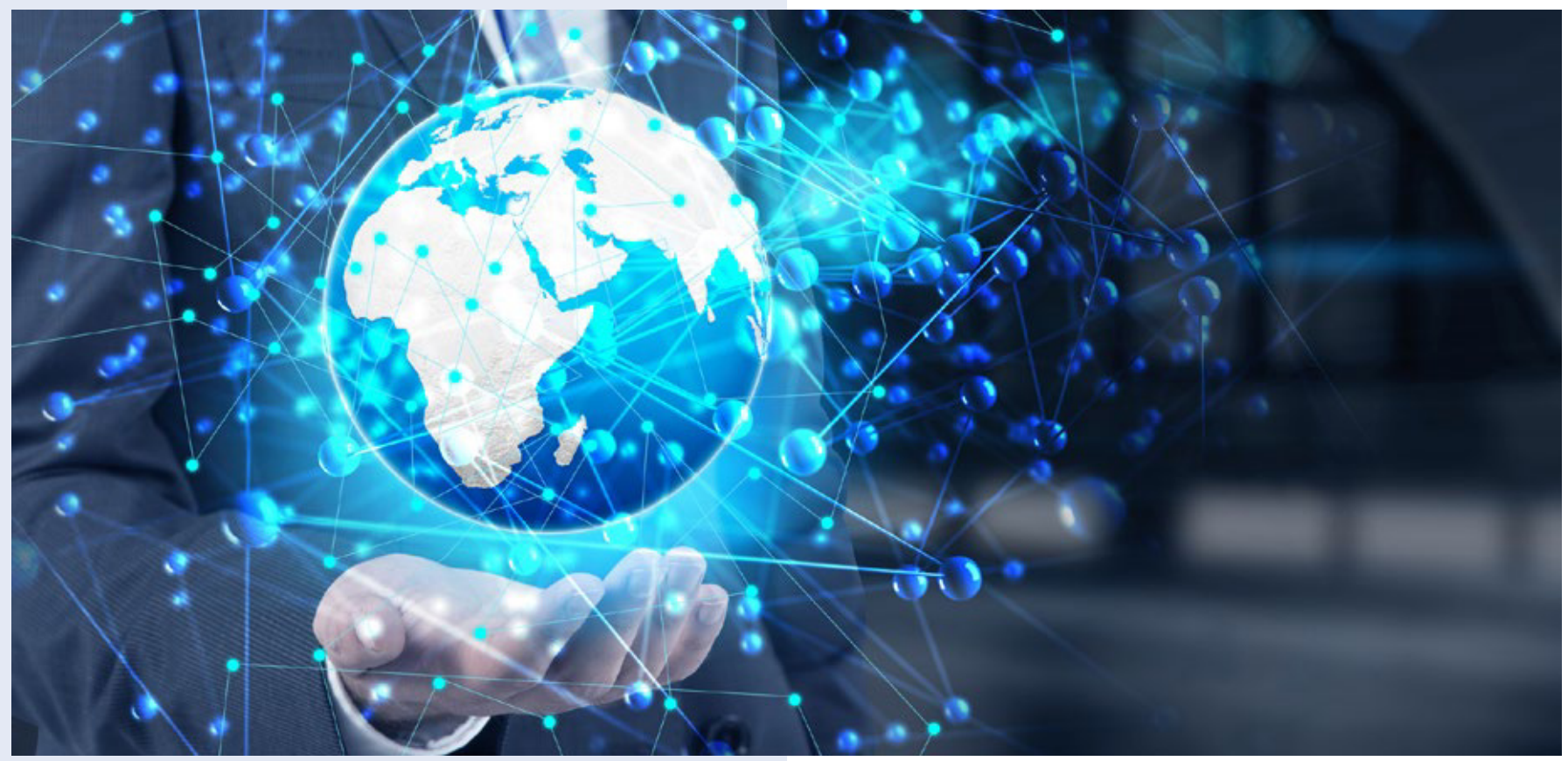



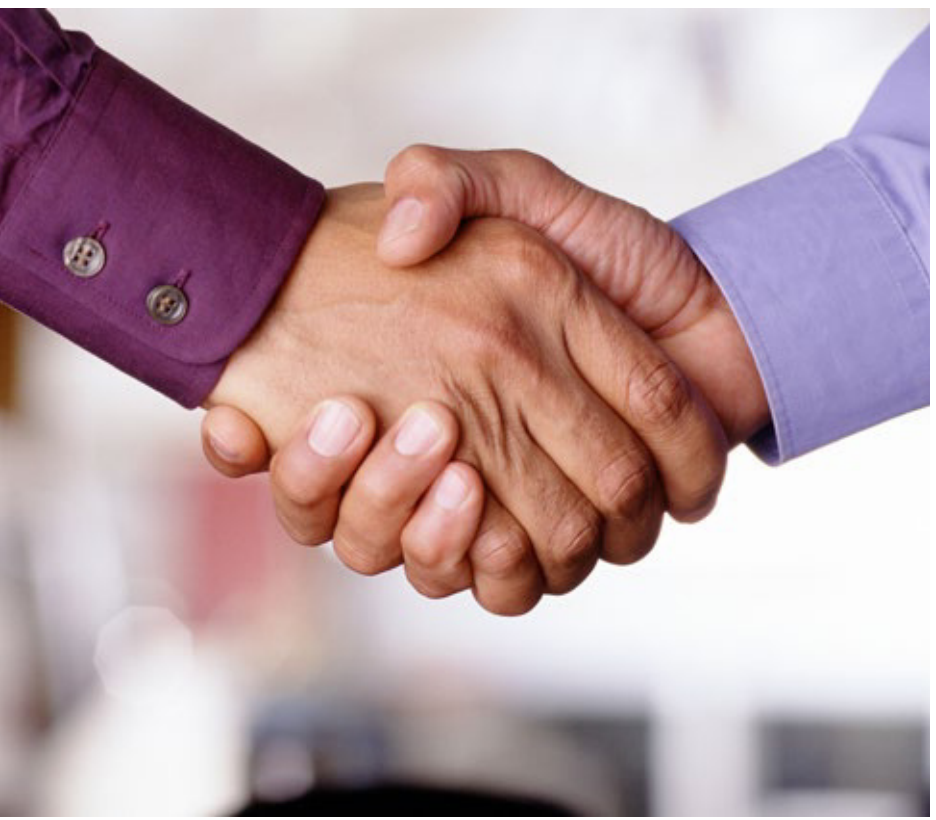

comunican con los ojos, con las manos, con los gestos, con la risa, con la palabra (Evertsz, 2000).

De acuerdo con la aseveración anterior y tomando en consideración la bibliografía consultada, cabe plantear que la comunicación puede manifestarse de tres formas: oral, escrita y no verbal. A su vez existen estudios que enmarcan la interpretación de la comunicación teniendo en cuenta los factores que inciden en ésta, dando como resultado la fórmula 7-38-55. El Dr. Albert Mehrabian, profesor de la Universidad de California en Los Ángeles, al analizar el impacto de un mensaje, llegó a la conclusión de que solo el $7 \%$ de la información que llega al receptor se atribuye a las palabras, mientras que el $38 \%$ se atribuye a la voz (entonación, proyección, resonancia, tono, matices, etc.) y el $55 \%$ restante al lenguaje corporal (señales, gestos, posturas, movimiento de los ojos, respiración. etc.).

\section{De esta manera, con C = Comunicación, tenemos:}

$C($ total $)=0,07 C($ verbal $)+0,38 C($ vocal $)+0,55 C($ lenguaje corporal)

Es importante referir que existen otras investigaciones que se oponen a esta asignación de índices, sin embargo, en general se coincide en la implicación del componente verbal y no verbal en el momento de la transmisión del mensaje. Pons, 2015, nos asegura que los gestos comunican sentimientos, emociones e intenciones; unas fracciones de segundo antes de que la persona inicie a hablar.

Entonces, teniendo en cuenta que la comunicación integra el componente verbal con el no verbal, para lograr una correcta interpretación del mensaje recibido, se debe combinar el resultado de la interpretación de ambos componentes de la comunicación. Ahora bien, usualmente se encuentra coherencia entre ambos componentes, pero si hay una desconexión entre lo que se está diciendo y lo que el cuerpo revela, entran en conflicto ambos mensajes (verbales y no verbales) generando ruido y distorsión en la interpretación de la comunicación recibida (Poyatos, 2013), (Stepke, 2012). Si se llegara a encontrar ante dicho conflicto, debe creer más en los mensajes no verbales, ya que, por lo general, provienen del inconsciente y son los más honestos, pero debe analizar dichos mensajes en su conjunto. Cada gesto, movimiento o cambio paralingüístico es una palabra individual que forma parte de una oración, y se deben interpretar las oraciones y no las palabras aisladas. Manejarlos en su conjunto, permitirá elaborar una imagen completa del mensaje (TarantinoCurseri, 2017).

Todas las relaciones interpersonales implican el proceso de comunicación, sería entonces, la negociación, un proceso que requiere de la comunicación bidireccional, y por ende la comunicación juega un rol determinante durante todo su ciclo de vida. Sin comunicación no hay negociación (Fisher, Ury y Patton, 2011); es por ello que resulta importante tener en cuenta los posibles errores que se podrían cometer en esta área (Manuel Dasí y Martínez, 2015):

\#o seguir el orden de una comunicación eficaz al negociar.

Wo realizar escucha activa a la hora de preguntar o responder.

\o adaptar de forma adecuada la comunicación.

» No reforzar la comunicación verbal con la no verbal.

Permitir que existan barreras a la comunicación en la negociación.

\No utilizar las ventajas que le ofrece escuchar para evitar los obstáculos en la negociación.

Por tanto, se puede concluir que en el momento de la presentación de la oferta las partes involucradas deben estar en estado de alerta; sobre todo teniendo en cuenta que en el proceso de comunicación existen elementos no verbales que en ocasiones comunican de forma más sincera y/o transparente el verdadero mensaje que subyace en el pensamiento de la contraparte negociadora.

Se proponen entonces las siguientes entradas, técnicas y herramientas, y salidas, para el desarrollo de la presente actividad (Figura 1).

\begin{tabular}{|l|l|l|}
\hline $\begin{array}{l}\text { Entradas } \\
\text { - Objetivos de la } \\
\text { negociación. }\end{array}$ \\
$\begin{array}{l}\text { - Estrategia de } \\
\text { negociación. } \\
\text { - Oferta. } \\
\text { - Plan de } \\
\text { presentación de la } \\
\text { oferta. } \\
\text { - Plan de gestión de } \\
\text { resgos. }\end{array}$ \\
\hline
\end{tabular}

Figura 1 Relación de entradas, técnicas y herramientas, y salidas de la actividad: Presentación de la Oferta. 


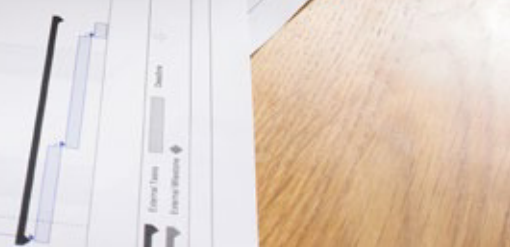

detallará las opciones que se hayan derivado de la actividad de elaboración de opciones.

\subsection{Técnicas y herramientas}

- Empleo de reglas de cortesía: en todo momento de interacción con la contraparte negociadora deberán emplearse elementos básicos de cortesía, con el fin de lograr un clima de respeto y confianza.

- Escucha activa: será utilizada con el fin de interpretar de mejor manera los objetivos y estrategias de negociación de la contraparte. Además contribuirá a incrementar la predisposición al diálogo y como consecuencia a entender mejor los planteamientos y a alcanzar acuerdos con mayor facilidad.

- Empatía: ayudará a situarse en la posición de la contraparte negociadora, con el fin de entender sus intereses y lograr la compatibilización con éstos.

- Técnicas de facilitación: serán empleadas para crear un ambiente de confianza y una comunicación fluida, que tribute a la prevención de conflictos y al alcance de acuerdos.

- Establecer criterios externos: a partir de éstos criterios se van a medir o comparar de forma justa, para las dos partes, las opciones. Cuando los demás factores son iguales, un acuerdo es mejor en la medida de que a cada parte le parece justo. Será justo para ellas en comparación con alguna referencia externa, algún criterio o principio. En este sentido pueden citarse como referentes externos de imparcialidad al valor de mercado, algún principio, el precedente, el derecho internacional, etcétera (Sepúlveda, 2012).

- Suba al Balcón (Ury, 1991): esta técnica consiste en salirse de la discusión y ver el asunto desde otra perspectiva; el objetivo es observar la negociación desde afuera y de esta manera ver cómo podemos hacer las cosas mejor.

- Replantee (Ury, 1991): esta técnica es útil cuando estamos tratando con contrapartes intransigentes, en cuyo caso sería de gran utilidad cambiar la perspectiva de la negociación, replantear la situación.

- Tienda un puente de oro (Ury, 1991): esta técnica propone no presionar a la contraparte para que tome una decisión, aconseja la creación de un ambiente de cordialidad donde se logren satisfacer necesidades de la contraparte como podrían ser: el reconocimiento, la seguridad, entre otras. Se debe lograr transmitir a la contraparte la satisfacción por el resultado de la negociación, lograr que sienta que obtuvo la mayor parte del triunfo en la mesa de negociación.

- Usar el poder para educar (Ury, 1991): esta técnica propone usar la persuasión para convencer a la contraparte de que uno busca lo mejor para ambas partes. Usar el poder para educar y para resolver las situaciones más complejas.

- Anclaje (Nocetti, 2007): esta técnica consiste en hacer una oferta inicial que logre influir en las percepciones de la contraparte negociadora sobre el conjunto de posibles resultados, de manera que ésta primera oferta actúe como ancla, cambiando la percepción que la otra parte tiene de su precio de reserva.

\subsection{Salidas}

- Resultados de la discusión de la oferta: relacionará todos los señalamientos o requerimientos de cambios o concesiones que haya realizado la contraparte negociadora. De igual manera relacionará los detalles que se consideren relevantes para la actividad de elaboración de opciones (Ejemplo: qué personas del equipo de la contraparte negociadora se mostraron de acuerdo con la oferta, quiénes se mostraron más reacios a su aceptación, etc.). Incluye la relación de acuerdos tomados durante la discusión de la oferta.

- Oferta: relaciona los elementos técnicos-comerciales de la propuesta, ajustados en correspondencia a los acuerdos tomados durante la discusión de la oferta.

\section{Actividad 3. Elaboración de opciones.}

Esta actividad será necesaria en caso de que no se hayan logrado acuerdos durante la discusión de la oferta, por lo que deberá realizarse un nuevo análisis por cada parte para la generación de opciones que ayuden al arribo a acuerdos. A partir de la obtención de nuevas opciones a negociar, se deberá proceder a realizar nuevamente la actividad de discusión de la oferta.

Se proponen las siguientes entradas, técnicas y herramientas, y salidas, para el desarrollo de la presente actividad (Figura 3).

\begin{tabular}{|l|l|l|}
\hline $\begin{array}{l}\text { Entradas } \\
\text { - Objetivos de la } \\
\text { negociación. }\end{array}$ & $\begin{array}{l}\text { Técnicas y } \\
\text { herramientas } \\
\text { - Estrategia de } \\
\text { negociación. } \\
\text { - Oferta. } \\
\text { - Recumentisis de } \\
\text { - Empión. } \\
\text { presentación de la la } \\
\text { oferta. } \\
\text { - Resultados de la } \\
\text { discusión de la } \\
\text { oferta. }\end{array}$ \\
\hline
\end{tabular}

Figura 3 Relación de entradas, técnicas y herramientas, y salidas de la actividad: Elaboración de opciones. 


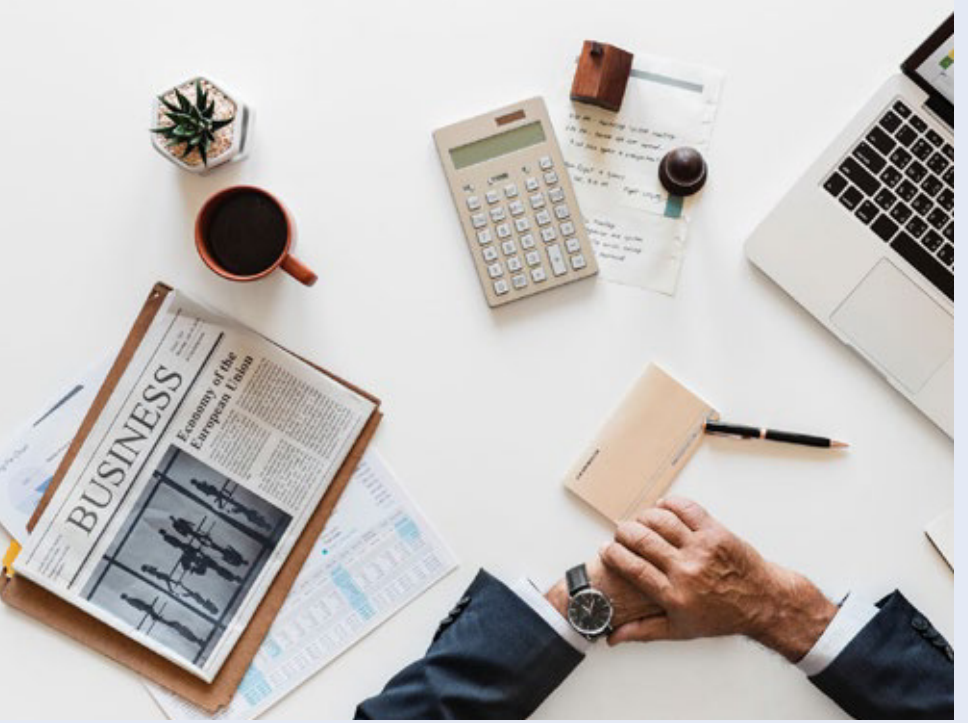

\subsection{Entradas}

- Objetivos de la negociación: establece claramente qué es lo que se quiere lograr con el proceso negociador.

- Estrategia de negociación: relaciona la información elaborada durante la etapa de planeación.

- Oferta: relaciona los elementos técnicos-comerciales de la propuesta.

- Resultados de la presentación de la oferta: este documento contendrá los principales elementos señalados durante la presentación de la oferta, así como los detalles que se consideren relevantes para la actividad de discusión de la oferta (Ejemplo: comentarios preliminares sobre la aceptación o no de la oferta, etc.)

- Resultados de la discusión de la oferta: relacionará todos los señalamientos o requerimientos de cambios o concesiones que haya realizado la contraparte negociadora. De igual manera relacionará los detalles que se consideren relevantes para la actividad de elaboración de opciones (Ejemplo: qué personas del equipo de la contraparte negociadora se mostraron de acuerdo con la oferta, quiénes se mostraron más reacios a su aceptación, etc.). Incluye la relación de acuerdos alcanzados.

\subsection{Técnicas y herramientas}

- Análisis de documentación: será enfocada al estudio de los documentos resultantes de la discusión de la oferta, de manera que sirva de base para la elaboración de nuevas propuestas de opciones.

- Empatía: ayudará a situarse en la posición de la contraparte negociadora, con el fin de entender sus intereses y lograr la compatibilización con éstos.

- Método de la 3ra alternativa: Se propone como primer paso analizar sus intereses propios, en segundo lugar valorar a la contraparte negociadora sin ningún pensamiento discriminatorio, luego poner en práctica la empatía para comprender a la contraparte, y finalmente lograr la sinergia, para lo que Stephen Covey plantea cuatro pasos: preguntar: ¿estás dispuesto a buscar una solución mejor?, definir los criterios del éxito para las dos partes, crear: experimentar con distintas soluciones y finalmente alcanzar terceras alternativas (Covey y England, 2012).

- Reuniones: se realizarán con el fin de aunar criterios sobre las propuestas de opciones.

- Juicio de expertos: a partir del análisis de las entradas, de la información arrojada de la aplicación de las técnicas anteriores, y basado en la experticia de los expertos; se propondrán diferentes opciones.

- Indicadores de factibilidad económica: se emplearán para determinar la factibilidad de las opciones propuestas.

\subsection{Salidas}

- Descripción de opciones a negociar: este documento contendrá el detalle de las nuevas opciones propuestas como variantes a la oferta que no fue aceptada inicialmente por la contraparte negociadora.

- Oferta: relaciona los elementos técnicos-comerciales de la propuesta, ajustados en correspondencia a las opciones propuestas durante el desarrollo de la presente actividad.

\section{RESULTADOS Y DISCUSIÓN}

La propuesta de guía fue validada mediante la aplicación de una encuesta a 20 expertos en el desarrollo de negociaciones. Dichos expertos se han desempeñado en el rol de especialistas y/o líderes por más de cinco años; estando vinculados a negociaciones, tanto comerciales como técnicas. Los resultados arrojados fueron los siguientes:

1. El $75 \%$ de los encuestados plantea que no cuentan con un procedimiento que les indique qué actividades realizar en la etapa de ejecución de una negociación.

2. El $91,67 \%$ de los encuestados plantea que no cuentan con una guía que les indique las técnicas y herramientas a utilizar en cada una de las actividades a desarrollar durante la etapa de ejecución de una negociación.

3. El $16,67 \%$ de los encuestados califica de regular la propuesta de actividades a desarrollar durante la etapa de ejecución de una negociación, el $41,67 \%$ considera buena la propuesta y el mismo porcentaje la considera muy buena.

4. El $58,33 \%$ de los encuestados califica de buena la propuesta de las técnicas y/o herramientas a emplear en la actividad de presentación de la oferta, mientras que el otro $41,67 \%$ la considera muy buena.

5. El $33,33 \%$ de los encuestados califica de buena la propuesta de las técnicas y/o herramientas a emplear 
en la actividad de discusión de la oferta, mientras que el otro $66,67 \%$ la considera muy buena.

6. El $41,67 \%$ de los encuestados califica de buena la propuesta de las técnicas y/o herramientas a emplear en la actividad de elaboración de opciones, mientras que el otro $58,33 \%$ la considera muy buena.

Además se recogieron las siguientes propuestas de elementos a incluir en la guía (ver Tabla 1).

\section{Tabla 1: Relación de propuestas a incluir en la guía, arrojadas por la aplicación de encuestas.}

\begin{tabular}{l|c|}
\multicolumn{1}{|c|}{$\begin{array}{c}\text { Elementos de la encuesta / } \\
\text { propuestas a incluir }\end{array}$} & \multicolumn{1}{c|}{$\begin{array}{c}\text { Cant. } \\
\text { Repeticiones }\end{array}$} \\
\hline Actividades propuestas & 4 \\
\hline 1. Selección de opciones & 1 \\
\hline 2. Aprobación/cancelación & \\
\hline Presentación de a oferta & 2 \\
\hline 3. Reconocimiento de emociones & 4 \\
\hline 4. Empatía & 1 \\
\hline 5. Retroalimentación & \\
\hline Discusión de la oferta & \\
\hline - Sin sugerencias & \\
\hline Elaboración de opciones & 1 \\
\hline 6. Oportunidades y posibilidades de clientes & 3 \\
\hline 7. Indicadores comerciales y sociales & 2 \\
\hline 8. Técnicas de Creatividad &
\end{tabular}

\section{CONCLUSIONES}

Mediante el desarrollo de la presente investigación se ha obtenido una propuesta de guía para la etapa de ejecución de las negociaciones, que servirá de base para el trabajo de los especialistas que desempeñen dichas funciones.

A partir del análisis de los resultados arrojados en la encuesta aplicada, se puede concluir:

1. La presente investigación es pertinente, ya que un alto porcentaje de los encuestados plantea no contar con un procedimiento que cubra la etapa de ejecución de las negociaciones, ni con una propuesta de guía que les indique las técnicas y herramientas a utilizar en cada una de las actividades de dicha etapa.

2. Más del $85 \%$ de los encuestados valora de buena o muy buena la propuesta de actividades a realizar durante la ejecución de las negociaciones.

3. El total de los encuestados considera buena o muy buena la propuesta de técnicas y herramientas a utilizar en la presentación de la oferta.

4. El total de los encuestados considera buena o muy buena la propuesta de técnicas y herramientas a utilizar en la discusión de la oferta.

5. El total de los encuestados considera buena o muy buena la propuesta de técnicas y herramientas a utilizar en la elaboración de opciones.

Además se recomienda incluir los siguientes elementos en próximas versiones de la guía:

1. La actividad de selección de opciones.

2. La técnica de empatía y el reconocimiento de las emociones en la presentación de la oferta.

3. Indicadores comerciales y sociales en la elaboración y selección de opciones.

4. Técnicas de creatividad en la elaboración de opciones.

\section{REFERENCIAS}

[1] Anthopoulos, L. \& Xristianopoulou, A. (Mayo de 2012). Negotiation Models for Managing Projects: a Review. En, Management of International Business and Economic Systems (MIBES-ESDO) 2012, Conferencia Internacional, Larissa, Grecia.

[2] Antonio, J., \& Barraza, V. (2017). Desarrollo de negociación. Citación: Unidad de Diseño Educativo. Zapopan, Jalisco: CTA-CUCEA, Universidad de Guadalajara.

[3] Cano, J. A., \& Baena, J. J. (2015). Tendencias en el uso de las tecnologías de información y comunicación para la negociación internacional. Estudios Gerenciales, 31(136), 335-346.

[4] Casal, J. P. V. (2016). Manual de negociación y resolución de conflictos: Define objetivos, resuelve incidencias y obtén resultados. Barcelona, España: Profit Editorial.

[5] Covey, S. R., \& England, B. (2012). La tercera alternativa: Para resolver los problemas más difíciles de la vida. Paidos.

[6] Evertsz, C. J. B. (2000). La comunicación efectiva. Instituto Tecnológico de Santo Domingo.

[7] Fisher, R., Ury, W., \& Patton, B. (2011). Getting to yes: Negotiating agreement without giving in. Penguin.

[8] Linares, A., Calderón, F., Donatti, K., Hernández, L. \& Martínez, J. (2009). Estrategias y tácticas de negociación en la comercialización. (Tesis de pregrado). Instituto Politécnico Nacional Escuela Superior de Comercio y Administración, México.

[9] Manuel Dasí, F., \& Martínez, R. M. V. (2015). Los cien errores en los procesos de negociación. ESIC Editorial.

[10] Mills, H. A. (2016). Negociar: el arte de ganar. Parkstone International.

[11] Nocetti, V. (2007). Fundamentos de negociación. Serie Documentos Docentes (SDD), 5(1), 2-24.

[12] Ogliastri, E. (1998). El estilo negociador de los latinoamericanos. Una Investigación cualitativa. Colombia Internacional, 1(41), 70-83.

[13] Pons, C. (2015). Comunicación no verbal. Editorial Kairós.

[14] Poyatos, F. (2013). La Comunicación No Verbal como asignatura en Filologías Clásicas y Modernas. Didáctica: Lengua y Literatura, 25, 231-257.

[15] Sepúlveda, D. P. (2012). La Negociación Cooperativa, una Aproximación al Modelo Harvard de Negociación. Revista Chilena de Derecho y Ciencia Política, 3(2), 253-271.

[16] Stepke, F. L. (2012). La conducta implícita en la relación bipersonal. Persona, (15).

[17] Tarantino-Curseri, S. (2017). Una breve pincelada sobre algunas áreas del saber necesarias para una negociación exitosa. Suma de Negocios, 8(17), 63-78.

[18] Ury, W. (1991). Supere el No: Cómo negociar con personas que adoptan posiciones obstinadas. Editorial NormaBogotá-1996.

[19] Zapiola, C. M. A. (2009). La negociación: un enfoque transdisciplinario con específicas referencias a la negociación laboral. Cinterfor/OIT. 